\title{
Helmut Kramer Justiz im Dienst des Angriffskrieges
}

Kaum haben sich kurz vor der Bundestagswahl die Unionsparteien und in ihrem Schlepptau SPD und FDP auf öffentlichen Druck dazu durchgerungen, ebenso wie die Linkspartei das Unrecht der Wehrmachtsjustiz beim Namen zu nennen und die „Kriegsverräter“ zu rehabilitieren, wird der Versuch unternommen, wieder eine eigene Militärgerichtsbarkeit einzuführen.

Angesichts schlimmer Erfahrungen im Kaiserreich war die Militärjustiz 1919 und, nach ihrer Wiedererrichtung durch die Nazis, im Jahre 1945 abgeschafft worden. Der 1956 ins Grundgesetz eingefügte Art. 96 hatte zwar schon die theoretische Möglichkeit einer Wehrstrafgerichtsbarkeit eröffnet. Angesichts des zu erwartenden öffentlichen Widerstandes scheute man aber schon die bloße Diskussion darüber. Dennoch machten sich bald nach Gründung der Bundeswehr Juristen im Bundesjustiz- und Bundesverteidigungsministerium in aller Heimlichkeit an die Planung einer eigenständigen Militärjustiz. In den Schubladen wurden fertig erarbeitete Gesetzentwürfe bereitgelegt, die für Deserteure und andere Beschuldigte einen drastisch verkürzten Rechtsschutz vorsahen, außerdem Eingriffsrechte des jeweils kommandierenden Generals als „Gerichtsherrn“ unseligen Angedenkens sowie die Aufstellung von Sondereinheiten ähnlich den Bewährungskompanien der Wehrmacht. Ebenso vor der Öffentlichkeit verborgen, selbst unter Kollegen verheimlicht, ließen sich die als künftige Militärrichter bereits vorgesehenen Juristen nach Sardinien und Kreta fliegen, um dort in simulierten Gerichtsverhandlungen mit Staatsanwälten, Richtern und Angeklagten ihre künftige Tätigkeit einzuüben. Neben Schreibkräften hatten sie auch Bücherkisten mit juristischen Kommentaren und Lexika in polnisch, russisch und tschechisch dabei. Als der Skandal durch eine „Panne“ (im Haushaltsplan der Bundesregierung von 1984 wurde ein verkappter Posten entdeckt) und durch das Buch „Kampfanzug unter der Robe“ von Ulrich Vultejus ans Tageslicht kam, musste die Planung abgebrochen werden.

\section{Strafrechtliche Privilegierung von Soldaten?}

Dass die alte Forderung nach einer Sondergerichtsbarkeit fürs Militär heute wieder aufkommt, ist kein Zufall in einer Zeit, da in den besetzten Ländern zunehmend die Zivilbevölkerung Opfer von Bombenangriffen wird. Denn in einem bewaffneten Konflikt hat die Bundeswehr die Strafgesetze und das humanitäre Völkerrecht (die Genfer Konventionen) zu beachten. Da möchte man der Gefahr einen Riegel vorschieben, dass militärisches Unrecht aufgeklärt wird, das man lieber unter den Teppich gekehrt sehen möchte. Der Bundeswehrverband unter dem Vorsitz von Oberst Ulrich Kirsch beklagte die „Rechtsunsicherheit“ der Soldaten und forderte für sie „einen ganz anderen Rechtsstatus“. Und unverhohlen rief der frühere Verteidigungsminister Franz Josef Jung mit der plumpen Forderung, „Soldaten sollten nicht mit staatsanwaltschaftlichen Ermittlungen konfrontiert werden", nach einer Justiz mit bloßer Feigenblattfunktion, denn, so Jung weiter, ein Strafverfahren gegen Oberst Georg Klein mit dem Ziel der Aufklärung des Massakers bei Kundus hätte „katastrophale Folgen“ für die Bundeswehr. 
Wenn die Militärbürokraten in der Politik „Rechtssicherheit“ und „Handlungssicherheit" verlangen, geht es ihnen unmissverständlich um die Ausstellung eines Freibriefs. In der Debatte über die grausame Verfolgung von Deserteuren, „Wehrkraftzersetzern“ und „Kriegsverrätern“ durch die Wehrmachtsjustiz zwecks Aufrechterhaltung der „Manneszucht“ beschäftigten sich die Medien und selbst die meisten Historiker nur am Rande mit einer anderen wichtigen Funktion einer Militärjustiz: der Aufgabe, militärische Übergriffe gegen die $\mathrm{Zi}-$ vilbevölkerung okkupierter Länder zu legitimieren.

Nach dem von hohen Militärjuristen der NS-Zeit formulierten „Barbarossa“Kriegsgerichtsbarkeitserlass sollte sich die Wehrmachtsjustiz einfach gar nicht um solche Übergriffe kümmern. Dies war eine wesentliche Voraussetzung für den millionenfachen Massenmord an der jüdischen und übrigen Zivilbevölkerung in Polen und der Sowjetunion sowie an der Ausrottung ganzer Dörfer auch in Griechenland und Italien.

Viele heutige Juristen würden sich wohl eine generelle Anweisung zur Niederschlagung von Verfahren wegen Ausschreitungen gegen die Zivilbevölkerung verbitten. Die historische Willfährigkeit der Wehrmachtsrichter lenkt jedoch den Blick auf Gefahren, in denen jede eigens für den Militärbereich eingerichtete Sondergerichtsbarkeit steht: dass der rechtliche Opferschutz vernachlässigt wird und Übergriffe gegen die Zivilbevölkerung ungeahndet bleiben. Auch US-amerikanische Militärjuristen haben Höchstleistungen in der Rechtlosstellung der Opfer vollbracht: vom Vietnamkrieg (Stichwort: My Lai) über den Irak-Krieg (Stichwort: Abu Ghraib) bis zum „Antiterrorkrieg“ in Afghanistan und in aller Welt (Stichworte: Guantanamo, Geheimgefängnisse der CIA). Und was geschieht mit den von deutschen Soldaten gemachten und an die Amerikaner übergebenen Gefangenen?

Solange die Tötung von Zivilisten und andere in Afghanistan angerichtete „Kollateralschäden“ im Halbdunkel blieben, war das Bedürfnis nach einem rechtsfreien Raum für Soldaten und Offiziere wenig aktuell. Das änderte sich mit der Bombardierung der beiden Tanklastzüge bei Kundus am 4. September 2009. Damit wurde erstmals einer breiten Öffentlichkeit bewusst, dass Zivilisten als Kriegsopfer des Schutzes durch die Justiz bedürfen. Die Aussicht, dass unabhängige Juristen sich mit diesem und künftigen Kriegsverbrechen beschäftigen könnten, weckte bei denen, die sich keine Beschränkungen beim Übergang von als Aufbauhilfe deklarierten Militäreinsätzen zu Kriegsoperationen auferlegen möchten, das Verlangen nach einer „einsatzfesten“ Justiz.

\section{Militärjustiz durch die Hintertür?}

Was nach den Erfahrungen der Bundeswehr bis vor kurzem noch als Tabu galt, wird jetzt vom Bundeswehrverband mit Nachdruck gefordert: die Wiedereinrichtung einer Militärjustiz. Denn es könne nicht sein, dass einem Soldaten wegen ziviler Opfer in Afghanistan der Prozess gemacht werde (s. taz vom 12.9.2009). Gemeint ist die vom Bundeswehrverband schon nach der Erschießung eines afghanischen Jugendlichen im Jahre 2006 angedachte „Militärgerichtsbarkeit mit Staatsanwälten, die mit in den Einsatz entsandt werden" (Süddeutsche Zeitung vom 21.7.2009), sozusagen eine embedded justice.

Angesichts der Vergangenheit der deutschen Militärjustiz werden solche frommen Wünsche nach einer förmlichen Rückkehr zu einer Militärjustiz mit fest in die militärischen Strukturen eingebundenem Personal zwar nicht alsbald durchsetzbar sein. Doch sind längst Lösungen im Gespräch, die darauf hinauslaufen, 
dass sich mit der weniger rechtlich als politisch heiklen Materie nur „zuverlässige“ Juristen beschäftigen. Man will eine Justizpraxis erreichen, die sicherstellt, dass die Auslandsaktivitäten der Bundeswehr vom Recht möglichst ungestört bleiben. Der Koalitionsvertrag der neuen Bundesregierung sieht die Einrichtung einer „zentralen Zuständigkeit der Justiz“ für Bundeswehrstrafsachen vor. Das zielte zunächst auf die nicht nur räumlich nahe dem Einsatzführungskommando der Bundeswehr in Geltow angesiedelte Staatsanwaltschaft Potsdam. Ob die nach Ansicht der Befürworter durch solche Spezialisierung zu erwerbende besondere Sachkunde auch eine unbefangene faire Überprüfung von Menschenrechtsverstößen und Kriegsverbrechen garantiert, ist ungesichert.

Gibt es aber nicht auch die Möglichkeit, derartige Verfahren von vornherein auf eine solche Ebene zu hieven, wo die Rücksichtnahme auf „Bündnisverpflichtungen “ und andere militärpolitische Belange sichergestellt ist? Auf diesen Einfall ist die Staatsanwaltschaft Dresden gekommen. Nach wochenlangem Zögern, ob sie für den Fall der Tötung einer großen Zahl von Zivilisten bei Kundus überhaupt ein Aktenzeichen vergeben sollte oder nicht, hat sie das Verfahren gegen Oberst Klein an die Bundesanwaltschaft in Karlsruhe abgegeben. Diese ist bei Verdacht eines Verstoßes gegen das Völkerstrafrecht im Rahmen eines bewaffneten Konflikts an erster Stelle zuständig. Inzwischen werden auch im Bundesjustizministerium Überlegungen angestellt, die Zuständigkeit des Generalbundesanwalts auf weitere Bundeswehrverfahren auszudehnen.

In den Augen derer, die politisch heikle Verfahren handverlesenen Juristen anvertraut sehen möchten, wäre das sicher schon von der Personalwahl her die optimale Regelung: Die Bundesanwaltschaft ist mit vom Bundesjustizministerium ernannten Juristen besetzt, die absolute Loyalität gegenüber der Bundesregierung garantieren. Damit kann die erwünschte Steuerung der Rechtsprechung noch besser erreicht werden als mit der Errichtung einer Sondergerichtsbarkeit für die Bundeswehr.

\section{Unabbängig oder willfäbrig}

Welch großen Wert die Zweite Gewalt auf eine willfährige Dritte Gewalt gerade in Bundeswehrsachen legt, ist am Beispiel der für Disziplinarverfahren gegen Soldaten zuständigen Wehrdienstsenate des Bundesverwaltungsgerichts offenkundig geworden. Wenn - wie im Fall des Massakers bei Kundus - die Dienstaufsicht nicht darum herumkommt, ein Disziplinarverfahren einzuleiten, etwa wegen Missachtung der ISAF-Regeln, wird in letzter Instanz einer der beiden Leipziger Senate damit befasst werden.

Nach dem Grundgesetz sind Richter unabhängig und nicht an Weisungen gebunden. Doch gerade im Fall der Leipziger Wehrdienstsenate ist die richterliche Unabhängigkeit in Frage gestellt. Eine auf den ersten Blick unauffällige, aber eindeutig verfassungswidrige Vorschrift ( $\$ 80$ Abs. 2 der Wehrdisziplinarordnung) ermöglicht es nämlich, diese Senate mit der Bundesregierung genehmen Juristen zu besetzen: Das Präsidium des Bundesverwaltungsgerichts darf die Wehrdienstsenate nur mit solchen Richtern besetzen, die das Bundesjustizministerium speziell für diese Aufgabe bestimmt hat.

Als würde dieser Eingriff in die Unabhängigkeit nicht genügen, hat nach einer in keinem Gesetzblatt stehenden Vereinbarung zwischen Justiz- und Verteidigungsministerium vom 21. Oktober 1970 das Verteidigungsministerium, also eine Prozesspartei, das Recht, die Richter für die Wehrdienstsenate auszusuchen. Diese Senate sollen als Werkzeug des Ministeriums dienen - das hat im September 
2009 der damalige Minister Franz Josef Jung bewiesen. Kaum war die Möglichkeit eines Disziplinarverfahrens gegen Oberst Klein, den Verantwortlichen des Bombardements bei Kundus, an den Horizont gerückt, lehnte Jung einen bereits vom Präsidium des Bundesverwaltungsgerichts in den für Klein zuständigen Senat gewählten Richter ab, dem der Makel anhaftete, nie in der Bundeswehr gedient zu haben: einen veritablen Wehrdienstverweigerer. Man verständigte sich dann auf einen anderen, dem Verteidigungsministerium genehmen Juristen. Inzwischen haben die Präsidenten des Bundesverwaltungsgerichts und aller Oberverwaltungsgerichte einhellig protestiert und die Streichung des $\$ 80 \mathrm{Abs} .2 \mathrm{der}$ Wehrdisziplinarordnung gefordert.

\section{Strafrecht als Freibrief für Massaker?}

Das verfassungsrechtliche Gebot der Unabhängigkeit gilt für die Richter, aber nicht für die Staatsanwälte, auch und erst recht nicht für den Generalbundesanwalt und seine Behörde. Dass zu den Qualitätsanforderungen an eine Behörde mit der Aufgabe, ebenso umstrittene wie robuste Militäreinsätze juristisch abzusichern, neben einer virtuosen Beherrschung der Rechtsauslegungskünste auch ein robustes Gewissen gehört, haben die zuständigen Beamten der Bundesanwaltschaft in den letzten Jahren wiederholt unter Beweis gestellt: So hat der Generalbundesanwalt nur die Vorbereitung eines Angriffskrieges, nicht aber den Angriffskrieg selbst für strafbar erklärt. Ein Ermittlungsverfahren gegen Donald Rumsfeld lehnte er mit der Begründung ab, um die Foltervorwürfe könne sich ja auch die US-Justiz kümmern. Mit der Verschleppung von Gefangenen in ausländische Geheimgefängnisse habe sich die CIA nicht strafbar gemacht, denn nur totalitären Staaten, nicht aber demokratischen Regierungen seien Verschleppungen verboten.

Was durfte man da erwarten, als sich der Generalbundesanwalt, Frau Monika Harms, wie angekündigt, mit den inzwischen eingetroffenen Unterlagen der Staatsanwaltschaft Dresden beschäftigt hat?

Gerade für Delikte wie die von Oberst Klein zu verantwortende Tötung von Zivilisten hatten trickreiche Militärjuristen bei der Formulierung des einschlägigen Straftatbestandes ( $\$ 11$ Abs. 1 Nr. 3 des Völkerstrafgesetzbuches) bereits Vorsorge getroffen: Das bei seiner Verabschiedung in den Medien hoch gelobte deutsche Völkerstrafgesetzbuch von 2002 lässt es nämlich nicht genügen, dass der „Angriff die Tötung oder Verletzung von Zivilpersonen in einem Ausmaß verursachen wird, das außer Verhältnis zu dem insgesamt erwarteten konkreten und unmittelbaren militärischen Erfolg steht". Vielmehr muss der Soldat oder Offizier diese Folge „als sicher erwarten“. Es sollte nicht einfach sein, eine solche Absicht nachzuweisen. Nach den gesamten Umständen hat Oberst Klein zwar die Tötung von Zivilisten in Kauf genommen. Ein solcher Fall wird von $\$ 11$ Abs. 1 Nr. 3 VStGB aber nicht erfasst und fällt damit aus der Zuständigkeit des Generalbundesanwalts heraus. Zuständig für nicht unter das Völkerstrafgesetzbuch fallende Tötungshandlungen wäre die gewöhnliche Staatsanwaltschaft, in diesem Fall wieder die Staatsanwaltschaft Dresden. Um das zu vermeiden, müsste der Generalbundesanwalt ein weiteres Begriffskonstrukt aus dem reichhaltigen Arsenal der Juristerei aufgreifen und $\mathbb{1 1}$ Abs. 1 Nr. 3 VStGB zu einer Spezialnorm des Kriegsvölkerrechts erklären, die weitergehende Straftatbestände des einfachen Strafrechts ,aufzehrt“. Dann hätte der Gesetzgeber mit $\mathbb{1 1}$ VStGB einen Freibrief für Kollateralschäden an Zivilisten ausgestellt, deren Eintritt nicht ganz sicher war. Die prompte Einstellung des Verfahrens gegen Klein hat diese 
Exklusivität des Völkerstrafgesetzbuches in der Eile noch nicht sicherstellen können.

Die von Barack Obama angekündigte „Erzwingung des Kriegsendes“ mit einer Verstärkung der Militäraktionen unter Beteiligung der Bundeswehr kann zu einer weiteren Eskalation des Krieges führen. Bei der zu erwartenden Häufung der „Kollateralschäden“ ist weder von der amerikanischen noch von der deutschen Justiz eine rechtliche Begrenzung zu erwarten. Der Satz „Wo kein Kläger, da kein Richter" ist ebenso banal wie sich seine Richtigkeit bewahrheitet, wenn willfährige Staatsanwälte am Werk sind.

\title{
Studien zum Strafrecht
}

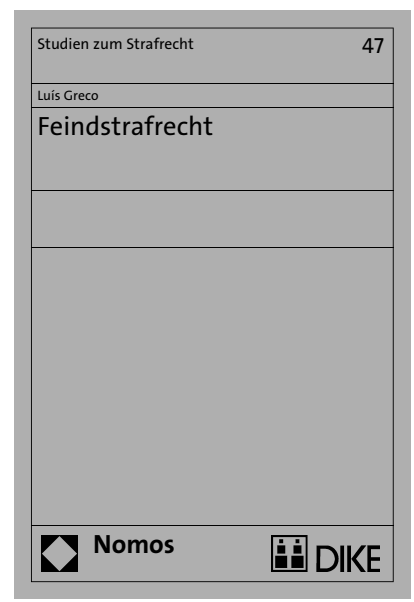

\author{
Feindstrafrecht \\ Von Dr. Luís Greco, LL.M. \\ 2010, Band 47, $98 \mathrm{~S}$., \\ brosch., 24,90€, \\ ISBN 978-3-8329-5799-5
}

Die Arbeit zieht eine Bilanz der weltweit außerordentlich heftig geführten Diskussion über das Feindstrafrecht. In Auseinandersetzung mit den wichtigsten für und gegen das Konzept angeführten Argumenten untersucht die Arbeit den Ertrag des Begriffs „Feindstrafrecht" für die Strafrechtswissenschaft. 